\title{
NOTAS
}

\section{FRECUENCIAS DE LA ASIBILACIÓN DE /R/ Y / RR/ EN MÉXICO}

En su nota sobre la "Distribución demográfica de la asibilación de vibrantes en el habla de la ciudad de México", publicada en este mismo volumen de la NRFH, pp. 71-79, G. Perissinotio estudia las frecuencias de asibilación de $/ \mathrm{r} / \mathrm{y} / \mathrm{rr} /$ en el dialecto de la ciudad de México, si bien, en el caso de / $/$, sólo toma en cuenta la posición final ante pausa. Me ha parecido interesante completar ese estudio con el de la asibilación de $/ \mathrm{r} /$ (en cualquier posición implosiva) y $/ \mathrm{rr} /$ en diferentes puntos de la geografía mexicana, en un intento de precisar la extensión nacional del fenómeno.

Navarro Tomás, después de mencionar la asibilación de /r/ en los grupos [tr], [pr], [kr] como fenómeno dialectal corriente en parte de Álava, Navarra, Rioja y Aragón, y muy extendido en Hispanoamérica, observa que "la presenciar de este fenómeno coincide con el uso de una $\mathrm{rr}$ ápicoalveolar fricativa, que se ponuncia asimismo con asibilación más o menos desarrollada" 1 . Pero ni él ni otros tratadistas describen la asibilación de [-r] implosiva.

El fenómeno ha sido documentado con mayor frecuencia (y con estudios más sistemáticos) en el español americano que en el peninsular2. D. L. CANFIELD, loc. cit., ha tratado de explicarlo históricamente:

1 Tomás Navarro Tomás, Manual de pronunciación española, $11^{\mathrm{a}}$ ed., Madrid, 1963, pp. 120 y 124.-A1 igual que Perissinotto, representaré con [r] la vibrante simple, con $[\mathrm{rr}]$ la múltiple, y $[r]$ y $[r r]$ sus variantes asibiladas.

2 Hay documentación para Nuevo México, México, Guatemala, Costa Rica, Colombia, Ecuador, Perú, Bolivia, Chile, Argentina, Uruguay y Paraguay. Cf. Peter Boyd-Bowman, "Sobre la pronunciación del español en el Ecuador", NRFH, 7 (1953), 226-227; Delos L. Canfield, La pronunciación del español en América, Bogotá, 1962, pp. 87-88; DANIEL N. CÁRDENAs, "The geographic distribution of the assibilated R, RR in Spanish America", Orbis, 7. (1958), 407-408, 411; y LuIs Flórez, La pronunciación del español en Bogotá, Bogotá, 1951, p. 233.-Merecen especial mención algunos lugares en que el fenómeno se puede calificar de muy frecuente, como Bogotá y la Argentina. "En Bogotá la articulación de la $r r$ es frecuentemente ápicoalveolar fricativa asibilada $[\ldots]$. Ordinariamente los mismos hablantes que asibilan la $r r$, asibilan también la $-r$ final de palabra (sobre todo ante pausa)"; y no importa el nivel sociocultural: "A toda clase de personas es común en Bogotá la pronunciación frecuente de una $r$ continua, más o menos sorda y asibilada en final de palabra ante pausa" (Flókez, loc. cit.). En cuanto a la Argentina, hay "dos tipos de $r$ múltiple $[\ldots]$, perfectamente diferenciados por todos: la $r$ vibrante, correcta, de Buenos Aires 
admite, con Navarro Tomás y Amado Alonso, que en España sólo se da en el norte, pero como de allí no vinieron sino "muy pocos de los primeros pobladores" de América, concluye que la "tendencia a la asibilación" fue introducida por inmigrantes llegados de la España septentrional en el siglo xix. D. N. CÁrdenas, art. cit., p. 410, aventura una explicación fonológica: en latín había una oposición de cantidad $[\mathbf{n}] \sim[\mathrm{nn}], \quad[1] \sim[1 \mathrm{l}], \quad[\mathrm{r}] \sim[\mathrm{rr}]$, mientras que en español "correcto" la oposición es de calidad en el caso de $[n] \sim[\tilde{\mathbf{n}}]$ y de $[1] \sim[\lambda]$, pero de cantidad en el de $[\mathrm{r}] \sim[\mathrm{rr}]$, de manera que la innovación cualitativa $[r] \sim[r r]$ restaura el equilibrio de las oposiciones. Finalmente, otros autores han dicho algo acerca del fenómeno en cuanto a sus manifestaciones más o menos frecuentes según la edad, el sexo o el nivel sociocultural del hablante ${ }^{3}$.

Por lo que se refiere a México, puede decirse que las referencias bibliográficas anteriores a la mencionada nota de Perissinotto son muy escasas, y a veces poco. precisas. Boyd-Bowman, que en 1948 no había registrado casos de $[\mathrm{rr}]$ en Guanajuato, la encontró en 1952, junto con la fricativa sorda, y tuvo "la impresión de que estas variantes no pasaban de ser propias de mujeres de las clases semiculta y culta"5. En su artículo de 1958, Cárdenas publica un mapa de las zonas de asibilación y vibración de $/ \mathrm{r} /$ en Hispanoamérica, en el cual la mayor parte del territorio mexicano figura como no investigada $a^{6}$. Uno de los espectrogramas que aparecen en el estudio de Alvar sobre Oaxaca "permite documentar $[r r]$ ensordecida y asibilada en posición final absoluta", y otro muestra $[r r]$ en posición inicial`. Dos estudios más recientes, el de Cárdenas sobre Jalisco y el de Lourdes Gavaldón sobre una zona de Coa-

y su zona de influencia, y la $r$ asibilada, dialectal, del interior", y en un mapa del tenómeno se ve que "la zona de la $r r$ asibilada es algo más extensa que la de la $r r$ vibrante" (BERTa Elfna VIDAL DE BATTini, "Extensión de la RR múltiple en la Argentina”, Fil, 3, 1951, 181-182) -Con una orientación estructural más definida, O. L. Chavarría-Aguilar, "The phonemes of Costa Rican Spanish", Lan, 27 (1951), p. 249, dice acerca de la asibilación en Costa Rica: "/R/ is an apico-alveolar groove spirant, tense and very fricative; it has two allophones, fully voiced and voiceless, the latter occurring only in utterance-final position".

3 "Probablemente la asibilación obedece a un rasgo innato del español amanerado o mujeril" (CANFIELd, op. cit., p. 88). La pronunciación $[r r]$ se da esporádicamente "entre mujeres mexicanas de la clase media o la clase alta" (ibid.). En Bogotá, [r] se asibila en mayor o menor grado ante [s] "en habla semiculta y aun en la familiar rápida y descuidada" (Flórez, op. cit., p. 201). En los lugares donde se da, la asibilación "is predominant among women and children" (CÁrdENAs, art. cit., p. 410).

4 Son frecuentes afirmaciones como ésta: "En la misma posición, en final de palabra, ante pausa, hemos oído $r$ asibilada entre mejicanos" (Fuórez, op. cit., p. 2l6) .

5 Peter Boyd-Bowman, El habla de Guanajuato, México, 1960, p. 80. Cf. ibid., p. 78: "Final y en el grupo $t r$, sólo asibilan la $r$ las mismas personas que asibilan la rr múltiple".

6 Cárdenas, art. cit., p. 409. Sólo aparecen marcados los estados de Yucatán y Campeche y parte del de Jalisco. En la p. 408 se precisa que en la región de Los Altos (Jalisco) -al igual que en el pueblo de Antonito (Nuevo México), en Bogotá y en la Sicrra del Ecuador- es muy frecuente una $r$ sorda y asibilada en posición tinal de silaba, cuando va seguida de una consonante sorda, especialmente $s$.

7 Manuel Alvar, "Algunas cuestiones fonéticas del español hablado en Oaxaca (México)", NRFH, 18 (1965-66), p. 367. 
huila, ponen de relieve la frecuencia de la asibilación, y el segundo de ellos da a entender que la aparición de $[-r]$ es menos frecuente que la de $[r r]^{8}$.

En resumen, la asibilación parece darse más a menudo en Hispanoamérica que en España; es un fenómeno relativamente reciente; influyen en él la edad, el sexo y el nivel sociocultural de los hablantes; faltan estudios sistemáticos sobre la asibilación en México; y si es verdad que en general pronuncian $[-r]$ las mismas personas que pronuncian $[r r]$ (cf., por ejemplo, lo que dice Navarro Tomás), esta afirmación no parece ser enteramente válida para México.

Con el presente trabajo trato de establecer en forma más o menos sistemática la frecuencia de la asibilación de / $\mathrm{r} / \mathrm{y} / \mathrm{rr} /$ en México. Mis materiales proceden del proyecto de delimitación de las zonas dialectales del país. Las investigaciones de este proyecto se están llevando a cabo por dos sistemas: el uso de un cuestionario (fruto de la experimentación de dos cuestionarios previos) y el análisis de cintas magnetofónicas que recogen muestras de habla espontánea ${ }^{9}$. Debido precisamente a este carácter espontáneo, he preferido basarme sólo en las cintas. En un cuestionario, la respuesta suele ser una simple palabra, y no siempre se puede encontrar de manera fidedigna el dato fonético que se busca. En las cintas, en cambio, no sólo se dispone de un corpus lingüístico muy amplio, sino que los fenómenos fonéticos, y en particular los de fonética sintáctica, afloran con toda naturalidad en un entorno de conversación corriente y espontánea.

El total de informantes es 380 (237 hombres y 143 mujeres). Los distribuyo en tres grupos generacionales: GG I (de 18 a 35 años), GG II (de 36 a 55) y GG III (de 56 en adelante). Distingo cuatro niveles socioculturales, de acuerdo con su preparación escolar, su afición a la lectura, su ambiente familiar, etc.: NSC 1 (hablantes cultos), NSC 2 (semicultos), NSC 3 (semianalfabetos) y NSC 4 (analfabetos).

8 Daniel N. Cárdenas, El español de Jalisco, Madrid, 1967, p. 41, ofrece un cuadro de $[-r]$ en posición final absoluta en el cual, si se suman las asibilaciones sordas y las sonoras, se obtiene un total de $43.65 \%$; dice que la rehilante fricativa sonora "persiste en varios lugares de Los Altos" (p. 43) y, haciendo suyo el juicio de S. L. RoBE, añade que "esta pronunciación (tal vez asibilada) se nota particularmente en la pronunciación de las mujeres" (p. 44). Por su parte, Lourdes Gavaldón, "Aspectos fonéticos de Múzquiz (Coahuila)", $A L M, 8$ (1970), 230-231, dice que "la $/ \mathbf{r}$ / final absoluta se pronuncia alternativamente en varias formas", una de las cuales, que se da "de cuando en cuando", y "en el habla de la generación joven sobre todo", es la "fricativa ligeramente asibilada"; la $/ \mathrm{rr} /$, por su parte, es a veces fricativa y a veces asibilada, y "con la misma frecuencia alternan estos dos fonemas en todas las clases sociales; sin embargo, tras nasal o lateral, es sistemática la aparición de [rr] asibilada $[\ldots]$, y en la generación joven se manifiesta claramente una preferencia por la asibilada".

9 Véase Juan M. Lope Blanch, “Las zonas dialectales de México. Proyecto de delimitación", NRFH, 19 (1970), 1-11. Hasta octubre de 1972 se ha trabajado en unas cien localidades, en cada una de las cuales se han hecho cuatro grabaciones y se ha llenado un minimo de tres cuestionarios. 


\section{Hombres}

Mujeres

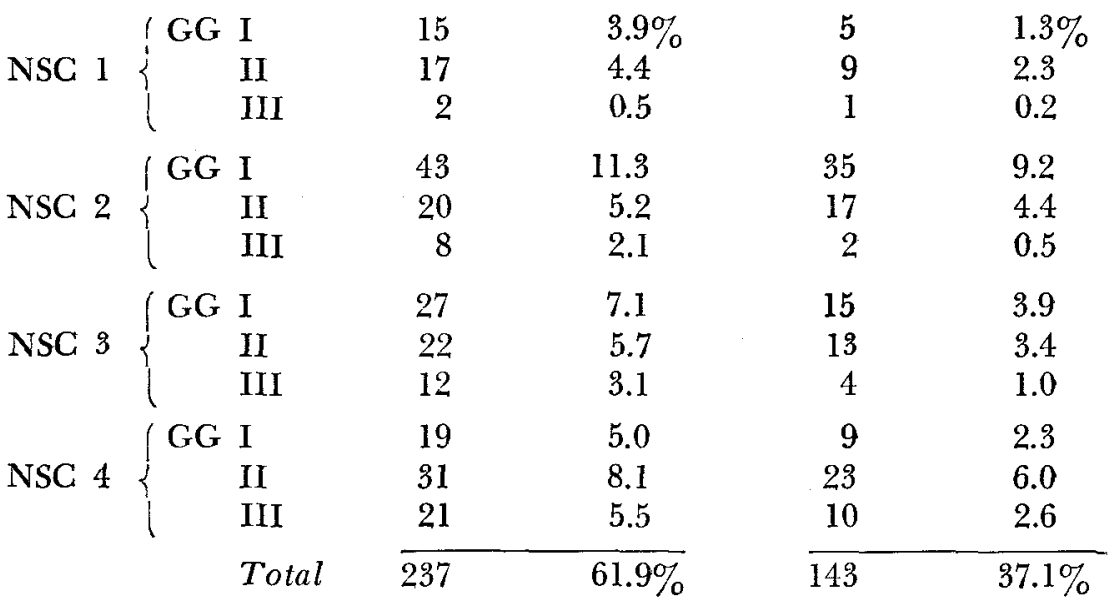

En los siguientes cuadros aparecen las frecuencias de asibilación tanto de / $/ \mathrm{r} /$ implosiva como de $/ \mathrm{rr} /{ }^{10}$. Para no complicar la comprensión de los cuadros he omitido otras especificaciones de tipo fonético, v.gr. la sonoridad o ensordecimiento, o el grado mismo de la asibilación. Establezco cinco grados de frecuencia: general (cuando se asibila casi el $100 \%$ de las / $/ \mathrm{r} / \mathrm{y} / \mathrm{rr} /$ ), frecuente (hacia el $70 \mathrm{u} 80 \%$ ), medio (hacia el $50 \%$ ), escaso (entre el 20 y el $40 \%$ ) y esporádico (menos del 20\%).

\section{Cuadro I. Frecuencla por nivel sociocultural}

\begin{tabular}{|c|c|c|c|c|c|c|c|c|}
\hline Grado de & NS & 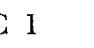 & NS & & & & & \\
\hline frec & {$[r]$} & {$[r r]$} & {$[r]$} & {$[r r]$} & {$[r]$} & {$[r r]$} & {$[r]$} & {$[r r]$} \\
\hline gene & - & - & $0.8 \%$ & $2.6 \%$ & - & $4.9 \%$ & $1.4 \%$ & 0.7 \\
\hline frecuente & $4.3 \%$ & - & $4.3 \%$ & $5.2 \%$ & $9.8 \%$ & - & $5.0 \%$ & $3.6 \%$ \\
\hline medio & $6.5 \%$ & $2.1 \%$ & $8.6 \%$ & $9.5 \%$ & $11.1 \%$ & $6.1 \%$ & $3.6 \%$ & $3.6 \%$ \\
\hline escaso & $10.8 \%$ & $4.3 \%$ & $13.0 \%$ & $12.1 \%$ & $9.8 \%$ & $7.4 \%$ & $6.5 \%$ & $7.2 \%$ \\
\hline esporádico & $6.5 \%$ & $15.2 \%$ & $10.4 \%$ & $6.0 \%$ & $6.1 \%$ & $9.8 \%$ & $7.2 \%$ & $5.7 \%$ \\
\hline
\end{tabular}

Cuadro II. Frecuencia por edades

\begin{tabular}{lcc|cc|cc} 
Grado de & \multicolumn{2}{c|}{ GG I } & \multicolumn{2}{|c}{ GG II } & \multicolumn{2}{c}{ GG III } \\
frecuencia & {$[r]$} & {$[r r]$} & {$[r]$} & {$[r r]$} & {$[r]$} & {$[r r]$} \\
\hline general & $0.5 \%$ & $1.1 \%$ & $0.6 \%$ & $2.6 \%$ & $1.7 \%$ & $3.4 \%$ \\
frecuente & $4.7 \%$ & $3.5 \%$ & $7.7 \%$ & $2.6 \%$ & $3.4 \%$ & $1.7 \%$ \\
medio & $7.1 \%$ & $5.7 \%$ & $9.1 \%$ & $7.7 \%$ & $1.7 \%$ & - \\
escaso & $8.8 \%$ & $9.5 \%$ & $8.4 \%$ & $8.4 \%$ & $15.6 \%$ & $5.1 \%$ \\
esporádico & $7.7 \%$ & $8.8 \%$ & $10.4 \%$ & $8.4 \%$ & $1.7 \%$ & $3.4 \%$
\end{tabular}

10 Debe tenerse en cuenta que la oposición fonológica $/ \mathrm{r} / \widetilde{\mathrm{rr}} /$ se neutraliza cuando $/ r /$ es final de sílaba: esto explica el tratamiento parecido de $[-r]$ y $[r r]$. (No tomo en consideración, para fines de porcentaje, la asibilación de los grupos [tr], [kr], etc., que, por lo demás, parece ser muy poco frecuente en México) . 
Como puede verse, la asibilación de la vibrante implosiva y de la vibrante múltiple, en los tres grados de frecuencia más importantes, muestra sus más altos porcentajes entre los hablantes semicultos y semianalfabetos. En los grados de frecuencia menos importantes, los porcentajes de asibilación de ambos alófonos son más homogéneos en los cuatro niveles socioculturales.

$\mathrm{Si}$ atendemos a la asibilación de /-r/ en los grados de frecuencia importantes, los porcentajes más altos corresponden al grupo generacional II, seguido del grupo I. Más o menos el mismo orden puede establecerse para la asibilación de /rr/. Sin embargo, en el grado genéral, el porcentaje más alto (algo engañoso a causa del bajo número de informantes) corresponde al grupo generacional más joven.

Cuadro ili. Frecuencia por sexos

\begin{tabular}{lrr|rr} 
Grado de & \multicolumn{2}{c|}{ Hombres } & \multicolumn{2}{c}{ Mujeres } \\
frecuencia & {$[r]$} & {$[r r]$} & {$[r]$} & {$[r r]$} \\
general & - & $1.6 \%$ & $2.1 \%$ & $2.8 \%$ \\
frecuente & $2.4 \%$ & $2.8 \%$ & $11.5 \%$ & $2.8 \%$ \\
medio & $2.4 \%$ & $4.5 \%$ & $15.2 \%$ & $7.9 \%$ \\
escaso & $10.7 \%$ & $7.8 \%$ & $7.9 \%$ & $9.4 \%$ \\
esporádico & $6.1 \%$ & $8.6 \%$ & $10.8 \%$ & $6.5 \%$
\end{tabular}

Es evidente que la asibilación de /-r/, en los grados de frecuencia importantes, se da en las mujeres mucho más que en los hombres. Son muy elocuentes los grados frecuente y medio, en que los porcentajes de asibilación femenina son cinco veces mayores que los de asibilación masculina. Para la asibilación de $/ \mathrm{rr} /$, en cambio, los porcentajes de asibilación son casi equivalentes en hombres y mujeres.

Cuadro IV. Frecuencia de la asibilación en general

$\begin{array}{ccc}\text { Grado de frecuencia } & {[r]} & {[r r]} \\ \text { general } & 0.7 \% & 2.1 \% \\ \text { frecuente } & 5.7 \% & 2.8 \% \\ \text { medio } & 7.1 \% & 5.7 \% \\ \text { escaso } & 9.7 \% & 8.4 \% \\ \text { esporádico } & 7.8 \% & 7.8 \%\end{array}$

Como se ve, la asibilación de / r/ es más frecuente que la de /rr/.

En resumen: la asibilación de $/-r / y / r r /$ en México es un fenómeno fonético poco documentado sistemáticamente, pero muy perceptible; hay que reconocer, sin embargo, que es (o es todavía) ${ }^{11}$ poco frecuente; se tiende a asibilar más la /-r/ implosiva que la / rr/ múltiple; definitivamente las mujeres asibilan más que los hombres, sobre todo la /-r/; el grupo generacional II asibila más que el I, y éste más que el III.

11 Puede preverse que el fenómeno va a adquirir mayor extensión y mayor frecuencia. En una investigación muy reciente (no tomada en cuenta aquí) que hicimos en la zona noreste del estado de Jalisco, pudimos observar que la asibilación es sumamente frecuente, más aún de lo que revela el libro de Cárdenas citado en la nota 8 . 


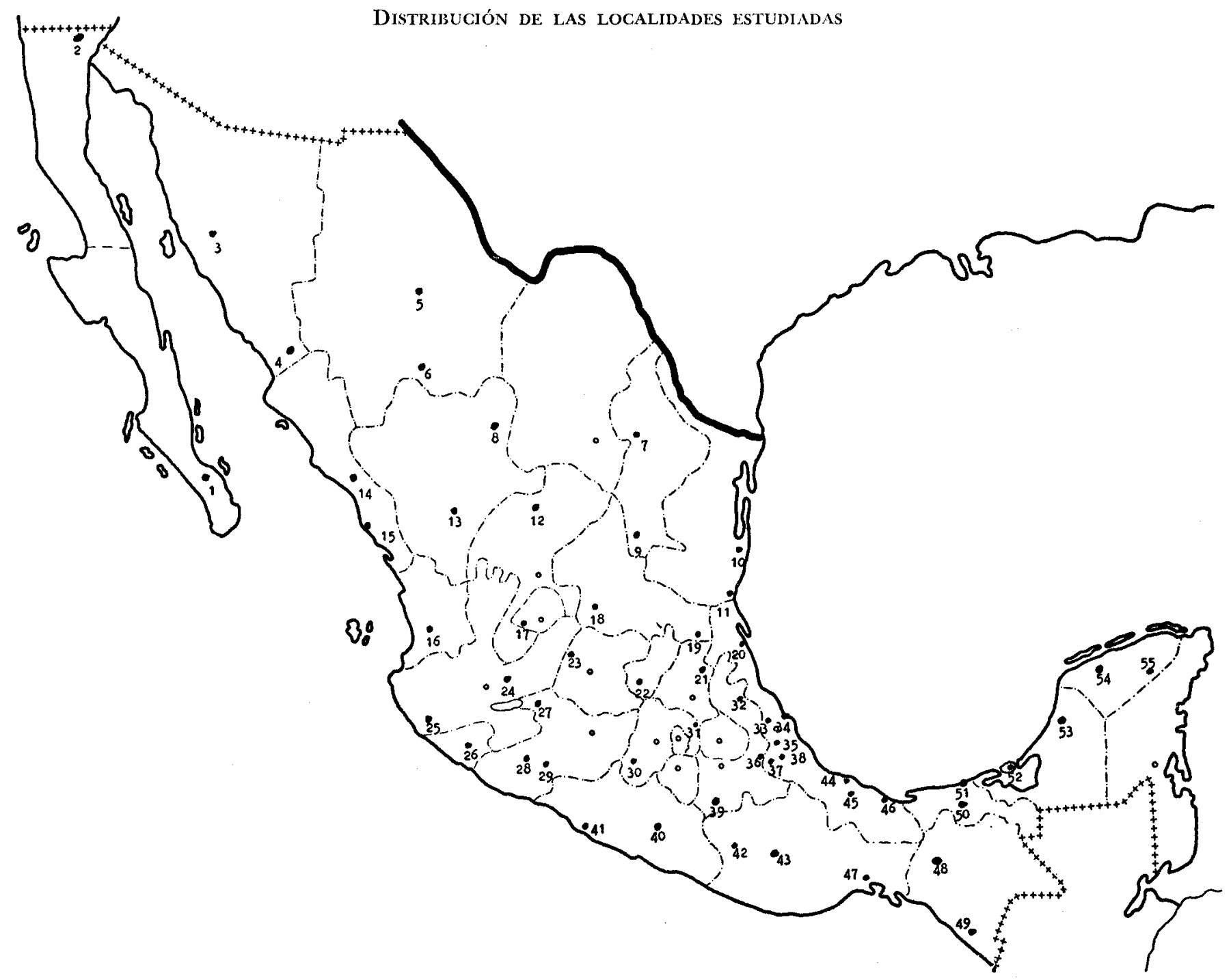


1. La Paz (Baja California)

2. Guadalupe Victoria (Baja California)

3. Hermosillo (Sonora)

4. Alamos (Sonora)

5. Chihuahua (Chihuahua)

6. Valle de Allende (Chihuahua)

7. Monterrey (Nuevo León)

8. Mapimí (Durango)

9. Doctor Arroyo (Nuevo León)

10 Soto la Marina (Tamaulipas)

11. Tampico (Tamaulipas)

12. Río Grande (Zacatecas)

13. Durango (Durango)

14. Culiacán (Sinaloa)

15. Mazatlán (Sinaloa)

16. Tepic (Nayarit)

17. Calvillo (Aguascalientes)

18. San Luis Potosi (San Luis Potosí)

19. Tamazunchale (San Luis Potosí)

20. Tuxpan (Veracruz)

21. Huasca (Hidalgo)

22. Querétaro (Querétaro)

23. León (Guanajuato)

24. Tepatitlán (Jalisco)

25. Villa Purificación (Jalisco)

26. Colima (Colima)

27. Zamora (Michoacán)

28. Apatzingán (Michoacán)
29. La Huacana (Michoacán)

30. Temascaltepec (México)

:1. San Martín Texmelucan (México)

32. Tetela de Ocampo (Puebla)

33. Perote (Veracruz)

34. Veracruz (Veracruz)

35. Huatusco (Veracruz)

36. Ciudad Serdán (Puebla)

37. Orizaba (Veracruz)

38. Córdoba (Veracruz)

39. Acatlán (Puebla)

40. Tixtla (Guerrero)

41. Tecpan (Guerrero)

42. Tlaxiaco (Oaxaca)

43. Oaxaca (Oaxaca)

14. Tlacotalpan (Veracruz)

45. Otatitlán (Veracruz)

46. Minatitlán (Veracruz)

47. Juchitán (Oaxaca)

48. Tuxtla Gutiérrez (Chiapas)

49. Escuintla (Chiapas)

50. Villahermosa (Tabasco)

51. Frontera (Tabasco)

52. Ciudad del Carmen (Campeche)

53. Campeche (Campeche)

54. Mérida (Yucatán)

55. Valladolid (Yucatán) 
En relación con la nota de Perissinotto mencionada al comienzo, puede notarse que el fenómeno de la asibilación -atendiendo a las realizaciones de /-r/ implosiva (en cualquier posición) y de $/ \mathrm{rr} /$ - es menos frecuente a nivel "nacional" que a nivel "capitalino", de manera que, desde un punto de vista geográfico, podría verse en la ciudad de México un foco irradiador del fenómeno; que éste se presenta más en el habla femenina que en la masculina, y más en los hablantes de los GG I y II que en los del GG III; y que, desde el punto de vista sociocultural, el nivel medio presenta el mayor grado de frecuencia.

Universidad Nacional Autónoma de México

José G. Moreno de AlbA y El Colegio de México.

\section{EL CANCIONERO ESPAÑOL (COD. REG. LAT. 1635) DE LA BIBLIOTECA VATICANA}

Presento en estas páginas la descripción y el índice detallado de un cartapacio desconocido de la segunda mitad del siglo xvi, como anticipo de la edición íntegra que de él estoy preparando. El ms., cuidadosamente ejecutado, contiene más de 230 composiciones de todo tipo, inéditas en su mayoría. Unos 145 textos (entre ellos un nutrido e interesante grupo de romances) ni siquiera se conocen en otros mss. El gusto muy amplio del recopilador permite considerar la colección como un buen ejemplo de las preferencias temáticas y formales de la época. Es oportuno anunciar el hallazgo del nuevo cancionero en estos momentos en que, gracias a los trabajos infatigables del malogrado Antonio Rodríguez-Moñino, se ha suscitado un interés tan grande por la poesía cancioneril del Quinientos. El nuevo ms., además de enriquecer el caudal poético del periodo, contribuirá a la fijación de textos críticos de poemas conocidos y aclarará muchos problemas de diversa índole, como atribuciones, influencia y cronología.

Se trata del Codex Reginensis Latinus núm. 1635 de la Biblioteca Vaticana ${ }^{1}$. Formó parte, evidentemente, de los antiguos fondos de la colección Reginensis, o sea la biblioteca particular de la reina Cristina de Suecia, que se constituyó a mediados del siglo xvir y pasó a la Vaticana en 1690. Sin embargo, el catálogo de esa colección, terminado en 1691 y publicado en 1739 , no incluye nuestro ms. entre los dos mil que enumera ${ }^{2}$. Es verdad que entre 1690 y 1740 "le fonds de la Reine

1 No lo he consultado directamente, sino en el microfilm que se guarda en la colección de la Knights of Columbus Vatican Film Library, Saint Louis University.

2 El catálogo fue publicado por Montraucon en las pp. 14-61 y 96-97 de la Bibliotheca bibliothecarum manuscriptorum nova (1739). He consultado Les manuscrits de la reine de Suède au Vatican. Réédition du catalogue de Montfaucon et cotes actuelles, Città del Vaticano, 1964 (Studi e testi, 238) : el núm. 1560 del catálogo (hoy núm. 81) se intitula "Anonymi Hispanica carmina", pero esto es errata por Hisperica Famina. (Véase la descripción de este ms. del siglo IX en ANDREas WILMART, Codices Reginenses Latini, t. 1, Bibliotheca Vaticana, 1937, p. 179.) 